\title{
Molecular basis for soft tissue management of gingivitis, periodontitis and peri-implant mucositis using an FDA cleared inflammation-targeting hydrogel - high potency polymerized cross-linked sucralfate - A case series illustrated profile of a drug device (Orafate) and the clinical rationale for use
}

\author{
Ricky W. McCullough ${ }^{1,2 *}$ \\ ${ }^{1}$ Translational Medicine Clinic and Research Center, Mueller Medical International, Storrs Connecticut, USA \\ ${ }^{2}$ Department of Medicine \& Emergency Medicine Veterans Administration Medical Center, Teaching Hospital, Warren Alpert Medical School of Brown University \\ Providence, Rhode Island 02908, USA
}

\begin{abstract}
Background: Current practice of soft tissue management (STM) targets the biofilm and its invasion into gingival soft tissue and periodontal space. Prior to FDA clearance of high potency polymerized cross-linked sucralfate (Orafate) there were not therapeutic options that targeted inflammation of the gingival and periodontal soft tissue structures. High potency polymerized cross-linked sucralfate (HPPCLS) for soft tissue management of oral inflammation represents an advance toward balanced medical management of oral inflammatory disease and wounds of oral surgery.

Aim: To profile an FDA-cleared sucralfate-based medical device, covering the dental proof of concept, its pharmacology, mechanisms of action and the clinical rationale of its use which is to target oral inflammation. To provide initial rationale for the use of Orafate as an adjunctive treatment of gingivitis, periodontitis and peri-implant mucositis.

Methods: Profile the clinical use of an inflammation-targeting hydrogel using a case series of 6 patients -severe periodontitis, deep pockets, post-cleaning dental pain, peri-implant mucositis, acute gingivitis, and dentin hypersensitivity. Review the concept and practice of STM and the pathophysiology of biofilm-induced soft tissue inflammation so as to provide the molecular basis of signs and symptoms of gingival inflammation. Review dental proof of concept to use sucralfate to target dental inflammation. Discuss the pharmacologic difference of HPPCLS and generic sucralfate.

Results: 1. Current STM options proactively target the biofilm with indirect attention to process of gingival inflammation. 2. All six patients responded rapidly to HPPCLS with complete resolution of signs ands symptoms of inflammation. 3. Molecular basis of dental pain centers on A-fiber inneration of dentinal tubules, and C-fiber innervation of dental pulp with either subject to infection or inflammation. 4. Soft tissue response to advancing biofilm is complicated process involving gingival defensins, activation of gingival dendritic and T-cells. 5. Gingival inflammation is not an open-ended event, but rather a feed-back controlled (genomiccontrolled) process of pro-inflammatory and anti- inflammatory cytokines. Historically, topical application of sucralfate has benefited mucosal inflammation when it occurs. 6. But HPPCLS is pharmacologically more potent than plain sucralfate on normal tissue by $800 \%$ and on inflamed tissue by $2,400 \%$ in terms of topical dose to the mucosa. 7. Case series illustrate the molecular basis by which HPPCLS rapidly resolves common clinical presentations.
\end{abstract}

Conclusions: Because HPPCLS targets inflammation, accelerates healing and rapidly reverses pain, it should be considered by dental practitioners as an adjunctive therapy for complete STM of gingival and periodontal health. The case series provide initial rationale of HPPCLS use as well as a spectrum of patients that may benefit from HPPCLS.

Practical Implications: In conjunction with current options, STM of mucosal inflammation may be aided by the inclusion of an FDA cleared sucralfate-based medical device containing HPPCLS.

\section{Introduction}

The molecular basis of traditional soft tissue management centers on removal of matrix-enclosed micro-organisms, known as plaque. Targeting the process of inflammation within the gingival soft tissue is hardly a focus, due in large part to the lack of inflammation-targeting therapeutic agents. Gingivitis, periodontitis and peri-implant mucositis are disorders of soft tissue inflammation, whose cause - invasive dentaladherent plaque, biofilm and bacterium - is more easily addressed. Recent FDA clearance of a hydrogel that targets inflammation presents
Correspondence to: Ricky W. McCullough, MD, MSc, Translational Medicine Clinic and Research Center, Mueller Medical International, Storrs Connecticut; Department of Medicine \& Emergency Medicine Veterans Administration Medical Center, Teaching Hospital, Warren Alpert Medical School of Brown University Providence, Rhode Island 02908, USA, Tel: 860-477-0961, E-mail: glencopelph@aol.com

Key words: gingivitis, peri-implant mucositis, sucralfate, HPPCLS, orafate

Received: May 16, 2017; Accepted: June 01, 2017; Published: June 05, 2017 
McCullough RW (2017) Molecular basis for soft tissue management of gingivitis, periodontitis and peri-implant mucositis using an FDA cleared inflammationtargeting hydrogel - high potency polymerized cross-linked sucralfate - A case series illustrated profile of a drug device (Orafate) and the clinical rationale for use

an opportunity to inculcate accelerated healing as an additional goal of traditional soft tissue management. Understanding the molecular basis of this hydrogel is the impetus for this report.

Disorders of gingival inflammation affect a vast majority of general population and lead ultimately to tooth loss. Disease prevalence is often assess using the Loe-Silness index of gingivitis [1,2], where 0 is no inflammation, 0.1-1 is mild inflammation (slight change in color, slight edema, with no bleeding on probing), 1.1-2 is moderated inflammation (redness, edema, glazing, bleeding on probing) and 2.1-3 is severe inflammation (marked redness, marked edema, ulceration with tendency of spontaneous bleeding). A reported population estimate of $50-100 \%$ for gingivitis [3] has been been characterized as having $94 \%$ with a gingivitis index of 0.5 or higher and $56 \%$ with an index of 1.0 or higher [4] with an overall average gingivitis index of 1.055 Elsewhere it has been reported [5] that over 50\% of adults had gingivitis on 3 to 4 teeth, that periodontitis with pockets $\geq 4 \mathrm{~mm}$ were present in $30 \%$ of population, severe pockets $\geq 6 \mathrm{~mm}$ were found in $4 \%$. Aattachment loss $\geq 3 \mathrm{~mm}$ was found in $40 \%$ of the population with gingival recession accounting for a significant amount of attachment loss. Peri-implant mucositis, or implant gingivitis, typically occurring 7-10 days following implant has an incidence of $80 \%$ in patients with $50 \%$ of their implant sites being affected [6,7]. Specifically, $48 \%$ of implants between year 9 and 14 are affected with mucositis [8].

Gingivitis, periodontitis and peri-implant mucositis are induced by plaque, fixed communities of periodontal bacterium. Thus, the therapeutic goal to reduced soft tissue inflammation, rides solely on efforts to eliminate the offending agent -plaque, which involves the use of antibiotics in conjunction with mechanical displacement of plaque. Therapeutic targeting of the process of inflammation within soft tissue (gingiva) has rarely been an objective for dental practitioners, such that to date, the standard of care for soft tissue management does not include therapies targeting soft tissue inflammation. As such there is no direct management of soft tissue inflammation. Though soft tissue inflammation is the primary problem, its resolution has been relegated to a secondary outcome resulting from the removal of invasive biofilm. It would be efficient and effective to do both - remove offending bioinvasive plaque while simultaneously accelerating the resolution of gingival irritation by targeting the process of soft tissue inflammation.

\section{Rate of Resolution of Inflammation vs Rate of Regrowth of Biofilm}

The rate of bacterial regrowth and its general resistance to antibiotics, easily overwhelms inherent soft tissue mechanisms tasked with restoration of homeostatic barriers. The latter are slow, indolent and sluggish. Patients undergo cleaning, scaling and root planning with some receiving either systemic or local delivery of antibiotics. Soft tissue inflammation, being untargeted, is left to both defend exposure to bacterial antigens at the dentigingival junction and simultaneously restore gingival barrier integrity. As an organ with a defensive role, the gingiva is never inactive. It cannot reduce continuous engagement of air, food and drink of various temperatures, consistency and mucoadherences, all of which substances interfere with the slow process of gingival healing.

This protracted nature by which gingival homeostasis is restored, while simultaneously defending against residual bacterial antigens, clearly disadvantages the patient. All too often, once gingival disease has advanced beyond preliminary stages, anti-plaque and antimicrobial therapies serve only to slow progression toward eventual and inevitable tooth loss. Due largely to poor patient compliance, restoration to baseline gingival health is challenging and often unlikely $[9,10]$.

\section{Targeting Plaque - Current Tools of Soft Tissue Management}

The stated goal of STM is to resolve the signs and symptoms of inflammation, reduce pocket depths to minimized attachment loss, and remove detectable plaque to a level consistent with periodontal health [11-13]. Nonsurgical therapy is the mainstay of periodontal care. Patient compliance, in-office detail and the adjunctive use of anti-infective treatments are the key elements. In the case of severe periodontitis, thorough periodontal debridement using a piezo or ultrasonic scaler under local anesthesia can halt disease progression. Scaling and root planing with hand instruments, together with inoffice irrigation using warmed medicaments such as chlorhexidine and fluoride and local delivery of antibiotic gels can also help arrest and some cases reverse disease. Low dose systemic antibiotics are gaining wider acceptance.

Locally delivered antibiotic agents include PerioChip (chlorhexidine), Arestin (minocycline) and PerioProtect (hydrogen peroxide), tetracycline fiber, metronidazole gel and doxycycline polymer. Motorized toothbrush use at home with frequent flossing and follow up biannual or quarterly cleanings complete an aggressive STM program [14].

In an emergency setting systemic antibiotics are preferred. Acute simple gingivitis due to streptococci, actinomycess or spirochetes are treated with either penicillin $\mathrm{G}$, amoxicillin-clavulante, or clindamycin. Necrotizing ulcerative gingivitis caused by provotialla intemidia, fusobacterium, tannerella forsythia, or treponema denticoli are treated with either metronidazole, amoxicillin-clavulanate, ampicillinsulbactam or clndamycin. Aggressive or localized juvenile periodontitis caused by actinobacillus, actinomycetemcomitans, porphyromons gingivalis, treponema denticola, or prevotella intermdidia is treated with either doxycycline or metronidazole. Adult periodontis due to treponema denticoli, poryphyromonas gingivalis, prevotella intermedia or tannerella forsythia require topical minocycline or doxycycline.

With each of these actions, whether routine or emergent, soft tissue inflammation is an indirect target. Actions focused exclusively on the tooth surface, associated plaque and invading bacterium can indeed reduce pro-inflammatory cytokine levels in crevicular fluid [15]. However, non-compliance - poor sustained oral hygiene regimen and missed returns for regular professional maintenance care - heavily influence undesirable therapeutic outcomes. An effective pharmacologic targeting of soft tissue inflammation would be a helpful tool for STM programs.

\section{Targeting Inflammation as a Therapeutic Option}

The real-world dynamics of slow healing inflamed gingiva and rapid regrowth of bacteria and plaque provide a clinical rationale for therapeutic options that exclusively target gingival inflammation. Short of medicinal options to accelerate healing and rapidly restore gingival defenses, anti-plaque therapies may be inadequate for some patients and could lead to surgery as their option of last resort to manage diseased and inflamed tissue [16].

Until recent, there have existed no pharmacologic options targeting ongoing inflammation within gingiva and periodontal structures. However, FDA approval of Orafate - high potency polymerized crosslinked sucralfate (HPPCLS) - was a step in that direction. Being the first pharmacological option exclusively targeting inflammation of gingival, periodontal and peri-implant soft tissue, HPPCLS represents an expansion of therapeutic options for soft tissue management in dental practice. HPPCLS was approved for use in-office immediately 
McCullough RW (2017) Molecular basis for soft tissue management of gingivitis, periodontitis and peri-implant mucositis using an FDA cleared inflammationtargeting hydrogel - high potency polymerized cross-linked sucralfate - A case series illustrated profile of a drug device (Orafate) and the clinical rationale for use

following cleanings, scaling and planning and at home in between cleanings and flossing. As such it offers an additional approach to gingival inflammation as an adjunct to anti-microbial treatments.

\section{Dental Proof of Concept for Sucralfate}

Dental proof of concept for using sucralfate as an oral antiinflammatory agent for the gingiva has been long established. Reported elsewhere, encrusted overlays (bulk topical dose) of generic sucralfate applied either as brushed-on paste or as a caked-dentifrice at the dental-gingival has been associated with the reversal of severe gingivitis and temporization of periodontitis in 30 to 180 days [17].

Its efficacy for gingivitis and periodontitis was anticipated by the use of HPPCLS in different patient populations, one wherein HPPCLS completely prevented and rapidly reversed gingival and mucosal ulcerations caused by chemoradiation in cancer treatment patients. In those patients, regardless of the clinical grade of oral ulcerative disease -Grade 1, minor, to Grade 4, severe - HPPCLS was associated with rapid (2-3 day) elimination of gingival and mucosal ulceration $[18,19]$. In this cohort of patients, the continued use of HPPCLS sustained the elimination of gingival and oral mucosal ulcerations despite continuous ongoing chemoradation. Beside this patient group, HPPCLS has been used to rapidly reverse aphthous and viral oral ulcerations as well as the inflammation of oral lichen planus. Given its efficacy in those inflammatory disorders of disparate etiologies, HPPCLS is likely to provide significant benefit to patients with gingivitis, periodontitis and peri-implant mucositis.

\section{Purpose of report}

The purpose of this report is to familiarize practitioners with the use of HPPCLS and its molecular and anatomical basis of action. A case series permits simultaneous illustration of HPPCLS use in various syndromes of gingival and periodontal inflammation and in turn provide a clinical backdrop to understand the molecular and anatomical basis of action of HPPCLS. The case series also allows for review of the inflammatory basis of signs and symptoms of oral disease commonly encountered in dental practice.

\section{Situational efficacy of HPPLCS}

Illustrating the situational efficacy of HPPCLS, reminds the clinician that gingival and periodontal inflammation is not 'a selflimited process' that quickly resolves once all measures against infection (scaling, planing, local or systemic delivery of antibiotics) have been optimized. Instead, while anti-infective measures are being optimized, the dental clinician can address gingival and pupal inflammation directly, separately and simultaneously. Thus, this device profile, illustrated by a six patient case series, seeks to furnish a better understanding of gingival inflammation and the molecular determinants contributing to the signs and symptoms of syndromes commonly encounter in clinical practice.

Following this case series is a review of known molecular determinants of perio-gingival inflammation (cytokines, growth factors, epithelial and immune cellular systems) and their role in gingival health and disease. The profile will then close with a discussion on the pharmacologic distinction between HPPLCS and generic (standard potency) sucralfate (which is neither polymerized nor crosslinked) and how its device mechanism of action lead to the improved clinical outcomes seen in the case series (Table 3). In this way, the report seeks to provide an initial rationale for HPPCLS use in the management of gingivitis, periodontitis and peri-implant mucositis.

\section{Device Profile - Orafate for Gingival and Periodontal Tissue Inflammation}

In contrast to historically slow resolution of non-targeted gingival inflammation, this six patient case series illustrates the rapidity and completeness by which Orafate works in the uncontrolled settings of every day clinical practice. The first set of three patients have periodontal disease- severe periodontitis maintained with non-surgical traditional treatments, chronic stabilized periodontal (deep pocket) disease and newly diagnosed mild periodontitis with post procedural gingival pain following "deep cleaning" by general dentist. The three patients of the second set have isolated dental issues - peri-implant mucositis, isolated acute aphthous gingivitis and apical infection associated dental pain (dentin hypersensitivity).

\section{Case series - Therapeutic breadth of HPPCLS}

\section{Case 1. Periodontal pain and tooth movement}

$52 \mathrm{yr}$ female with long-standing severe periodontitis involving the right maxillary central incisor, its lateral incisor and cuspid treated with scaling, planning and local delivery of antibiotic, developed bleeding, pain and looseness of $1^{\text {st }}$ incisor. She was treated with local application of Orafate $(0.8 \mathrm{ml})$ twice daily for 7 days. Pain and bleeding resolved in 2 days. Firmness of tooth restored in 4 days. Anticipated surgical intervention was averted for the time being.

\section{Case 2. Chronic moderate periodontal disease}

$56 \mathrm{yr}$ male followed by periodontist for 1 year development of $6 \mathrm{~mm}$ pocket depth involving the left mandibular $1^{\text {st }}$ and $2^{\text {nd }}$ molars with pain and bleeding on probing was treated over the past 12 months with intermittent local delivery of antibiotics, scaling and planning. $\mathrm{He}$ was treated with of Orafate $(0.8 \mathrm{ml})$ and swish and expectorate regimen twice daily for 2 weeks prior to return visit to periodontist. Pocket Depth improved to $4 \mathrm{~mm}$ with no pain or bleeding upon probing. Patient was instructed to continue Orafate daily.

\section{Case 3. Acute persistent gingival \& dental pain following "deep cleaning"}

$62 \mathrm{yr}$ female healthy gingiva followed by dentist with biannual dental cleaning for 10 years. Following a session of "deep cleaning" procedure by the dental hygienist, she developed gingival burning and palpable pain, and intermittent bleeding during procedure at the right mandibular $2^{\text {nd }}$ bicuspid and $1^{\text {st }}$ molar. The bleeding improved but persisted 5 minutes following procedure but gingival burning and palpable tenderness persisted and increased 2 hours post-procedure. There was pain in $2^{\text {nd }}$ bicuspid itself. Treatment with Orafate $(0.8 \mathrm{ml})$, dabbing onto site of pain and bleeding were met with immediate relief of dental pain and of gingival burning with diminished palpable gingival tenderness. The Orafate treatment was repeated in 2 hours. By hour three there was no bleeding and palpable tenderness had stopped. By the sixth hour with no bleeding and no palpable tenderness, there was a faint notice of gingival burning and of bicuspid pain, approximately $1 / 4$ the initial intensity post cleaning. An additional application of Orafate $(0.8 \mathrm{ml})$ was met with immediate cessation of gingival burning and bicuspid pain. Though gingival burning, palpable tenderness and bleeding had completely resolved, patient was advised to brush twice daily with Orafate for 1 week.

\section{Case 4. Peri-implant mucositis}

A 61 yr male with a dental implant of the right maxillary central incisor that was 16 years old. He developed peri-implant gingival pain 
McCullough RW (2017) Molecular basis for soft tissue management of gingivitis, periodontitis and peri-implant mucositis using an FDA cleared inflammationtargeting hydrogel - high potency polymerized cross-linked sucralfate - A case series illustrated profile of a drug device (Orafate) and the clinical rationale for use

and soreness with scant bleeding and pain on probing. The gingival hard palate at the implant site was treated initially with Orafate $0.8 \mathrm{ml}$ mixed with petroleum jelly $(0.8 \mathrm{ml})$ to facilitated Orafate adherence to peri-implant mucosal every 12 hours. Pain and soreness was relieved by $4^{\text {th }}$ application, but applications were continued for 2 additional days for a total of 4 days. Patient continued twice daily brushing with Orafate with no recurrence of the problem six months from its initial occurrence.

\section{Case 5. Acute gingivitis}

A 62 yr male with Type 2 diabetes with known recurrent periogingival mucositis, noted gingival point tenderness, induration and bleeding of the gums during flossing between the left maxillary lateral incisor and cuspid. Normally this episode would last 9-10 days before spontaneously subsiding. At the onset of this episode he was treated with $0.8 \mathrm{ml}$ Orafate mixed with similar quantity of petroleum jelly to the gingiva every 8 hours. Bleeding upon flossing stopped in 1 day, induration improved by $50 \%$ but point tenderness on flossing improved gradually over the next 24 hours with complete resolution of all symptoms by day 3 , less than half the time for spontaneous resolution.

\section{Case 6. Dental pain to sweets and cold temperature and pressure sensitivity}

A 63 yr male, with known enamel erosion, and nocturnal teeth grinding, developed 1 week of pain, followed by pressure pain (noted on chewing) and cold temperature sensitivity to his right mandibular $2^{\text {nd }}$ bicuspid which had a fractured, dental caries and in the right maxillary 1st molar with no previous dental injury. He was advised to brush twice daily with Orafate, starting with the affected teeth. By day 2 of the brushing, pain and cold temperature sensitivity had subsided. By day 4 pressure- pain sensitivity resolved. Two days after stopping Orafate brushing, patient reported return of the pressure pain and faint cold temperature sensitivity. He resumed twice daily brushing with Orafate by dabbing first the affected intradental gingival area then the entire gingival surfaces. Pressure pain and cold sensitivity resolved after 2 days and patient continued Orafate brushing for 1 month.

\section{Molecular Basis of Periogingival Inflammation}

Each patient of the case series illustrates some form of periogingival inflammation. Between bacterial invasion and inevitable connective tissue breakdown and tooth loss is the progression of gingival inflammation that can manifest itself as in any of the above case scenarios. Activated host defenses produce and release proinflammatory cytokines that result in tissue destruction. However, receiving far less attention are anti-inflammatory cytokines acting as feedback control of aggressive pro-inflammatory cytokines, which are under genomic control as well.

Anti-inflammatory cytokines are the second half of a two-part evolutionary process tasked with restoring homeostasis - that is, normal gingival health. In fact, it is best to think of normal gingival health as a controlled stand-off between invasive bacteria with chemical toxins and immuno-epithelial cells engaged in surveillance and barrier protection - a protection that is facilitated by constitutive secretion of molecular agents and the strategic expression homeostatic receptors engaged in signaling to maintain balance. This balance is considered normal gingival health.

Soft tissue reaction to bacterial assault involves a gradation of responses that are under genomic control. Each graded response is characterized by the release of feedback controlled pro-inflammatory cytokines that activate lamina proprial cells in a process that becomes are increasingly destructive. Fully inflamed gingiva is rendered a defensive maze of indurate tissue that seeks to stalemate advancing bacterium, though unsuccessfully so. While the determinants of periogingival inflammation begin with bacteria in antibiotic resistant plaque or the biofilm as their means to extend virulence, it eventually include resident and recruited cellular components of the gingiva aggressively engaging bacteria that seek to advance from periodontal plaque into the subepithelium of the gingiva.

To better understand, just how HPPCLS works, that is, its molecular and anatomical basis of action, each component involved in soft tissue inflammation will be discussed.

\section{The Dentigingival Junction}

Either side of the dentigingival junction - the dental and the gingival side - harbor respective landscapes of molecular items that drive the resultant process of soft tissue inflammation. On the dental side is the biofilm with the micro-architecture of the tooth, its functional components and their relationship with bone and the neurovascular bundle. On the gingival side is the epithelial barrier populated by epithelium, keratinized and non-keratinized, intraepithelial lymphocytes, dendritic cells and cells of the lamina propria.

\section{Dental side of inflammation - the biofim, the tooth and its functional structures}

Periodontal biofim: Gingivitis, periodontitis and peri-implant mucositis are biofilm mediated diseases. Unlike cellulitis, erysipelas, or soft tissue abscess, where a single organism dominates the site of infection intimately juxtaposed to the infected tissue, a biofilm is a community of disparate micro-organism, embedded within impenetrable matrix of polymers secreted by organisms and used as a mechanism to advance invasion into adjacent host tissue. As polymeric homelands of bacterial communities, plaque is polymer-enclosed nutrient-sufficient colonies of micro-organisms that release planktonic cells to colonize surfaces newly acquired by extrusion of adherent polymers produced by the community of bacterial cells.

Resistance to antibiotics is due impart to decreased penetration into plaque matrix [20] necessitating mechanical removal of plaque by scaling, planing, and brushing. Individually, biofilm cells can resist antibiotics by presenting molecular targets differing from those on planktonic cells that colonize newly secreted adherent polymers [21]. Also, biofilm cells develop efflux pumps to expel antimicrobials that breach their cellular membrane. As a whole, without physical removal of plaque, bacterial communities embedded in plaque resist local or systemic delivery of antibiotics [22,23].

Mapping of biofilm communities by fluorescence microscopy [24] reveals that matrix-embedded bacteria have a cell to cell distance of 4 to 5 micrometers. The planktonic bacterium first to colonized newly adherent polymeric matrix, will later adopt a phenotype within the matrix that does not permit growth even if plated on agar [25]. Direct studies have shown that organisms causing periodontitis grow almost exclusively in biofilms [26-28].

The chronic bacterial infection of the gingival crevice that causes periodontitis is a mixed-species biofilm, inherently resistant to antibiotics by matrix resistance to penetration and bacterial adaptation of molecular markers and efflux pumps. 
McCullough RW (2017) Molecular basis for soft tissue management of gingivitis, periodontitis and peri-implant mucositis using an FDA cleared inflammationtargeting hydrogel - high potency polymerized cross-linked sucralfate - A case series illustrated profile of a drug device (Orafate) and the clinical rationale for use

The Tooth and its Functional Structures: The tooth, sectioned into the crown above the gingival line and the root below it, is covered by enamel directly beneath which lies the dentin that extends from the crown inferiorly into the root and completely encases a central lying dental pulp, which is living connective tissue and odontoblastic cells with their pre-dentin composites. The following review, taken from the literature [29-32] provides an anatomical and physiological backdrop in understanding how Orafate may work.

Dentin: The dentin is comprised of centripetally oriented tubules, 0.5 to 2 micrometers in diameter, densely packed, that radiate away from the dental pulp toward the outer surface of the tooth in all directions. Proximally, at the pulpal interface, the dentinal tubules contain cytoplasmic extensions of odontoblasts (Tomes fibers), which though derived from the neural crest are responsible for dentin formation. Beyond these cytoplasmic extensions is fluid filling the dentinal tubules, with the distal end of the tubule covered with enamel coronally and with cementum on the root surfaces.

At the crown peripheral dentin is covered with enamel, hydroxyapatite, a mineral form of calcium phosphate embedded in matrix proteins, chiefly amelogenin. While the enamel is considered impermeable, small cations (potassium), water and hydrogen peroxide have low but significant permeation.

Pulp and Vasculature: Within dental pulp of connective tissue and odontablasts is the neurovascular bundle conducting arterials into the pulp ascending coronally toward the apex, branching into capillaries that service the odontoblasts and connective tissue, emerging as venules that in turn descend down the pupal root to join the alveolar vein associated with that tooth. The hydrostatic pressure within the pulp of $45 \mathrm{~mm} \mathrm{Hg}$ at the arterial portion of the capillary decreases to $35 \mathrm{~mm} \mathrm{Hg}$ mid capillary exiting at the venule portion of the capillary at $20 \mathrm{~mm} \mathrm{Hg}$. All the while hydrostatic pressure within the dentinal tubules ranges from $5-10 \mathrm{~mm} \mathrm{Hg}$. The capillary permeability, intracapillary osmolality and the differential pulpal pressure permit diffusion of nutrients and other macromolecules.

Neurovascular Bundle: The neurovascular bundle contains ascending afferent neurons of two classes, myelinated and nonmyelinated fibers. Myelinated A fibers, subdivided into A- $\beta$ and A- $\delta$, ascend toward the upper two thirds of the tooth and peripheral to the pulp, $40 \%$ of them innervating dentinal tubules above the pupal horns decreasing at the cervical dentin, then least represented in root dentin at only $3.5 \%$. The A- $\beta$ fibers are located near the cell body of the odontoblast at the pulp-dentin border and are stimulated at a low threshold as compared to A- $\delta$ myelinated fibers which extend beyond the body of the odonoblasts into dentinal tubules up to 150 microns. It is the A- $\delta$ myelinated fibers that are activated by hydrodynamic stimuli - cold air (vs heat), sweets, drilling, probing or hypertonic solutions. Hydrodynamic stimuli create rapid fluid flow within tubules. The pain from A-myelinated fibers is fast, sharp, short and well localized.

The non-myelinated C-fibers occupy the deeper central core of the pulp respond to heat (vs cold), mechanical deformation and inflammatory mediators. The pain from unmyelinated C-fibers is slow, dull, poorly localized and lingering. If a pain stimulus persists then additional $\mathrm{C}$-fiber neurons are recruited and the pain becomes a burning sensation and may refer pain into adjacent teeth. In anoxia environments within the dental pulp (infarct or poor blood flow), A-fibers, being larger and metabolically more demanding, will cease working, while C-fibers will continue to function. The molecular basis of the thermal reaction of these fibers is as follows: sudden application of cold and not heat cause fluid within the dental tubules to contract stimulating A-fibers but not C-fibers. Continued cold application, equilibrating fluid movement in tubules, result in diminished firing of A-fibers and decreased pain. Application of heat to a vulnerable tooth, will affect core of dental pulp where $\mathrm{C}$-fibers reside, leading to $\mathrm{C}$-fiber firing. Continued heat results in continued C-fiber mediated pain. Electric pulp testing causing shifts in electrolytes in tubule fluid will stimulate A-fibers, but not C-fibers. Percussive tapping of tooth that has an inflamed core generally will not escalate pain sensation; however as central papal inflammation extend toward the external periphery of the dentin where there is an incresase concentration of proprioceptors, then percussive tapping accentuate the pain response. Unmyelinated C-fibers are responsive to capsaicin, histamine and bradykinin, the fibers containing neuropeptides such as substance $\mathrm{P}$, calcitonin gene related peptide (CGRP) and neuokinin A.

Cementum and Periodontal Ligaments: Similar to osteoblasts that secrete bone and then become entrapped to become osteocytes, cementoblasts, likely originating from Hertwig's epithelial root sheath, secrete calcified material around the root of the tooth, then become entrapped by hydroxyapatite and are rendered as cemantocytes, lying with lacunae. Cementum is slightly softer than dentin containing $45 \%$ hydroxyapatite vs $55 \%$ hydroxyapatite in dentin.

The inorganic material is held by a matrix of collagen and proteoglycans. Being avascular the cementum receives nutrients from the vascularized periodontal ligament. Completely enveloping the dentin and extending coronally, the cememtum ends at the enamel, the neck or cervical region of the tooth. Collagenous fibers extend from the cememtum into the periodontal ligament (Sharpey fibers) and from the periodontal ligament into alveolar bone, thus anchoring the root of the tooth into alveolar bone.

Dotted along the periodontal attachments and adjacent to cememtum are additional vestiges of Hertwigs epithelial root sheath, known as the epithelial cell rests of Malassez. Epithelial cell rests of Malassez are thought to be associated with maintaining the integrity of the periodontal ligaments. The periodontal ligament contain fibroblasts, osteoblasts, cementoblasts as well as the epithelial cell rests. Cell rests of Malassez are believe to maintain periodontal ligament homeostasis, prevent ankylosis, preserve ligament space and serve as a target during periodontal ligament innervation as well as contribute to cementum repair when required.

\section{Gingival side of inflammation}

Epithelium, $\gamma \delta$ T-cells, Toll-like Receptors, Defensins, Dendritic Cells, PAMPS and DAMPS: Unlike the epithelial cells associated with the tooth formation, gingival epithelium is a adaptation of pre-existing oral mucosa accommodating to the submucosal eruption of dentition. As such, in health and disease, the gingival epithelium retains the homeostatic tendencies of the buccal mucosa.

Homeostatic barrier to invading bacteria is an active process supported by constitutive supply of regenerating epithelium, intraepithelial lymphocytes, saliva concentration of trefoil factors, and growth factors.

Gingivitis and periodontitis begin with biofilm-mediated assault of the dento-gingival junction. Left unabated, the inflammatory process proceeds to disrupt periodontal attachments and erode periodontal cementum. Lastly progressive inflammation affects alveolar bone by activating focal bone resorption and remodeling. Dendritic cells, T-cells and gingival epithelium expressing toll-like receptors (TLR) 
McCullough RW (2017) Molecular basis for soft tissue management of gingivitis, periodontitis and peri-implant mucositis using an FDA cleared inflammationtargeting hydrogel - high potency polymerized cross-linked sucralfate - A case series illustrated profile of a drug device (Orafate) and the clinical rationale for use

that process PAMPS (pathogen associated molecular pattern antigens) and trigger initial epithelial secretion of defensins (microbiocidal agents) and eventual secretion of damage associated molecular pattern particles (DAMPS) comprise the gingival homeostatic barrier that counteracts invasive biofilm.

Upper and lower part of gingival pocket: The morphology of crevicular and outer epithelium has been well defined histologically by Takarada et al. [33-35]. Figure 1 illustrates three distinct histopathological areas of the epithelial pocket in periodontitis [33]. The gingival crest is the transitional area of para-keratotic epithelium, lateral and distal to which sits fully keratinized epithelium and inferiomedial and distal to which sits a non-keratinized epithelium that trends toward keratinization as it extends upward toward the crest lying in an apical position. Juxtaposed to the tooth surface the crest of transitionally keratinized epithelium marks the top of the upper zone of pocket epithelium. The gingiva then dives downward and medially to a sub-zone of non-keratinized epithelium considered the lower part of pocket epithelium. In gingivitis, periodontitis and peri-implant mucositis, there is an abrupt change at the interface of the upper part and lower part of the pocket, a changed characterized by the presence of three zones of inflammation within the lower part of the gingival pocket. A gradient of inflammation most intense in the epithelium is at the periodontal surface, and it fans out away from the tooth extending into the subgingival tissue stroma or lamina propria.

The Soft Tissue Reaction - the Sequence of Events: Long before the arrival of large numbers of neutrophils to the site of plaque invasion with the associated tissue destruction, a series of graded immunological events transpire on the surface of the gingiva interfacing the biofilm. Existing mucosal biosurveillance is an active process involving the epithelial cells, their neighboring intraepithelial T-lymphocytes, and submucosal dendritic cells recruited by both to assess the microbial threat posed by the biofilm. It is into this environment that Orafate is introduced and it is within these events that its mechanism of action begins (Figure 2).

Gingival Intraepithelial Lymphocytes and Epithelial Immune Response: The gingival mucosa is constantly exposed to a wide range of organisms, some commensal, others pathogenic. Critical immune responses maintain the balance between elimination of harmful pathogens and calm coexistence with communal organisms.

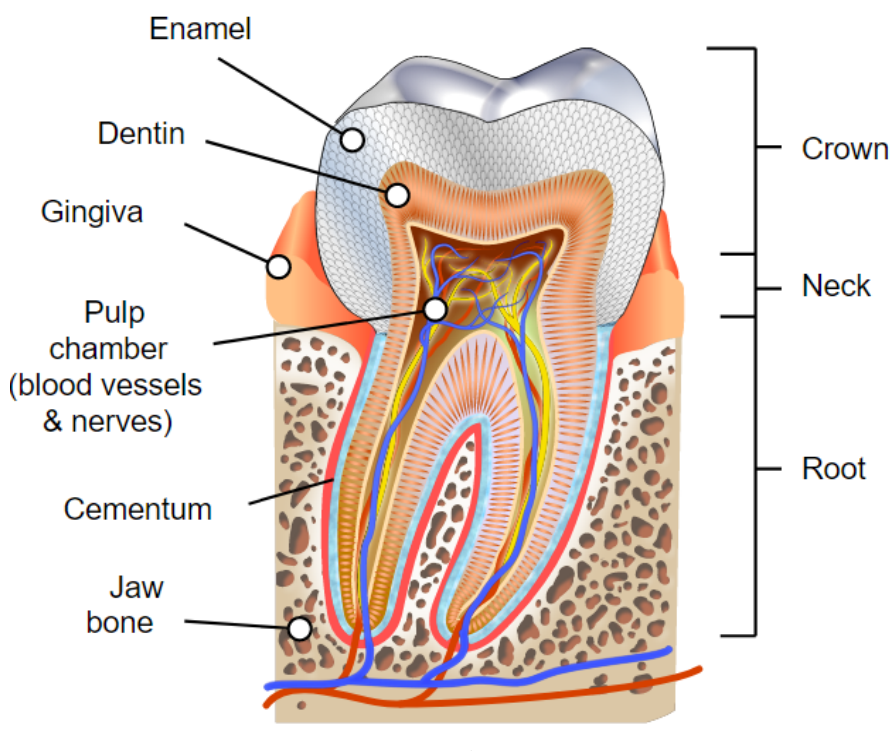

Figure 1. Tooth structure.
Gingival epithelium express toll-like receptors (TLR) that are capable of engaging and deciphering the pathogenicity of antigens shed by micro-organisms. Antigens identified as non-commensal prompt epithelial elaboration of defensins, which are anti-microbials capable of damaging the cell walls of unfriendly bacteria [36,37]. Simultaneously, TLR signals subepithelial dendritic cells in the lamina propria to protrude cytoplasmic extension beyond the epithelium to sample antigens for determination of virulence. Dendritic cells embody a separate level of threat perception and can opt to continue with the innate immune response involving epithelial elaboration of greater concentrations of defensins or upgrade the response by activating the adaptive immune response with the secretion of antigen-specific antibodies [38,39], the latter of which recruits involvement of lamina proprial lymphocytes, macrophages, and monocytes.

Defensins are antimicrobial molecules divided into two categories, alpha-defensins of which there are six types and beta-defensins of which there are four types. Beta-defensins are associated with human epithelial surfaces including gingiva.

When an antigen is perceived as a serious threat, signaling from the epithelial TLR triggering the expression of beta defensins which in turn leads to recruitment of subepithelial dendritic cell to sample the offending antigen and prompts the distressed epithelial cell to upregulate its neighboring intra-epithelial T-cells [40] to mount a more robust front line of defense that could be further escalated if required.

The gamma-delta intra-epithelial T-lymphocyte ( $\gamma \delta$ T-IEL) show preferential homing to the gingival epithelium and reside there as part of gingiva epithelial surveillance. In chronic periodontitis, $\gamma \delta$ T-IELs are upregulated to secrete IFN- $\gamma$, TNF- $\alpha$, TGF- $\beta 1$ and IL- $6[41,42]$. Additionally, distressed epithelial cells express IL-8, a chemotaxic agent for neutrophil infiltration.

Once neutrophils are present there is a graded escalation of focal inflammation, with feed-back controlled secretion of a-defensins, pro-inflammatory cytokines, chemotactic recruitment of monocytes, macrophages and destruction of bacterial cells and of epithelial cells and the subsequent release of pathogen-associated molecular patterns (PAMPs) [43] and damage-associated molecular pattern molecules (DAMPS) [44]. It is in this setting that progressive periogingival and periodontal tissue destruction occurs if significant feed-back signaling from the area of most intense inflammation is not transmitted to the nuclei of bordering epithelial gingival cells.

\section{Pharmacologic Distinction Between HPPLCS and Generic (standard potency) Sucralfate}

Orafate was cleared by the FDA for the management of oral inflammatory lesions and processes both in-office and at-home, to alleviate pain and facilitate the healing process by its muco-adherent ability to protect the mucosal lining against further irritation. Orafate contains high potency polymerized cross-linked sucralfate, or HPPCLS, which is standard sucralfate reformulated by polymerization with acid then cross-linked by cations that are in turn secondarily chelated (Table 1). The exact configuration of sucralfate within HPPCLS is unknown, but it is believed that singular molecular sucralfate is crosslinked by multi-valent cations into linear sheets of sucralfate that resist the van der Waal forces of hydration. These 'sheets of sucralfate' are then polymerized by multi-dentated acidic chelators. Polymerizing electronegative sheets of sucralfate formed by cationic cross-linkages of individual sucralfate molecules facilitates an orderly, compounded layering of sucralfate on the mucosal lining, in 'pancake' fashion a process known as pi-(П) stacking $[45,46]$. 
McCullough RW (2017) Molecular basis for soft tissue management of gingivitis, periodontitis and peri-implant mucositis using an FDA cleared inflammationtargeting hydrogel - high potency polymerized cross-linked sucralfate - A case series illustrated profile of a drug device (Orafate) and the clinical rationale for use

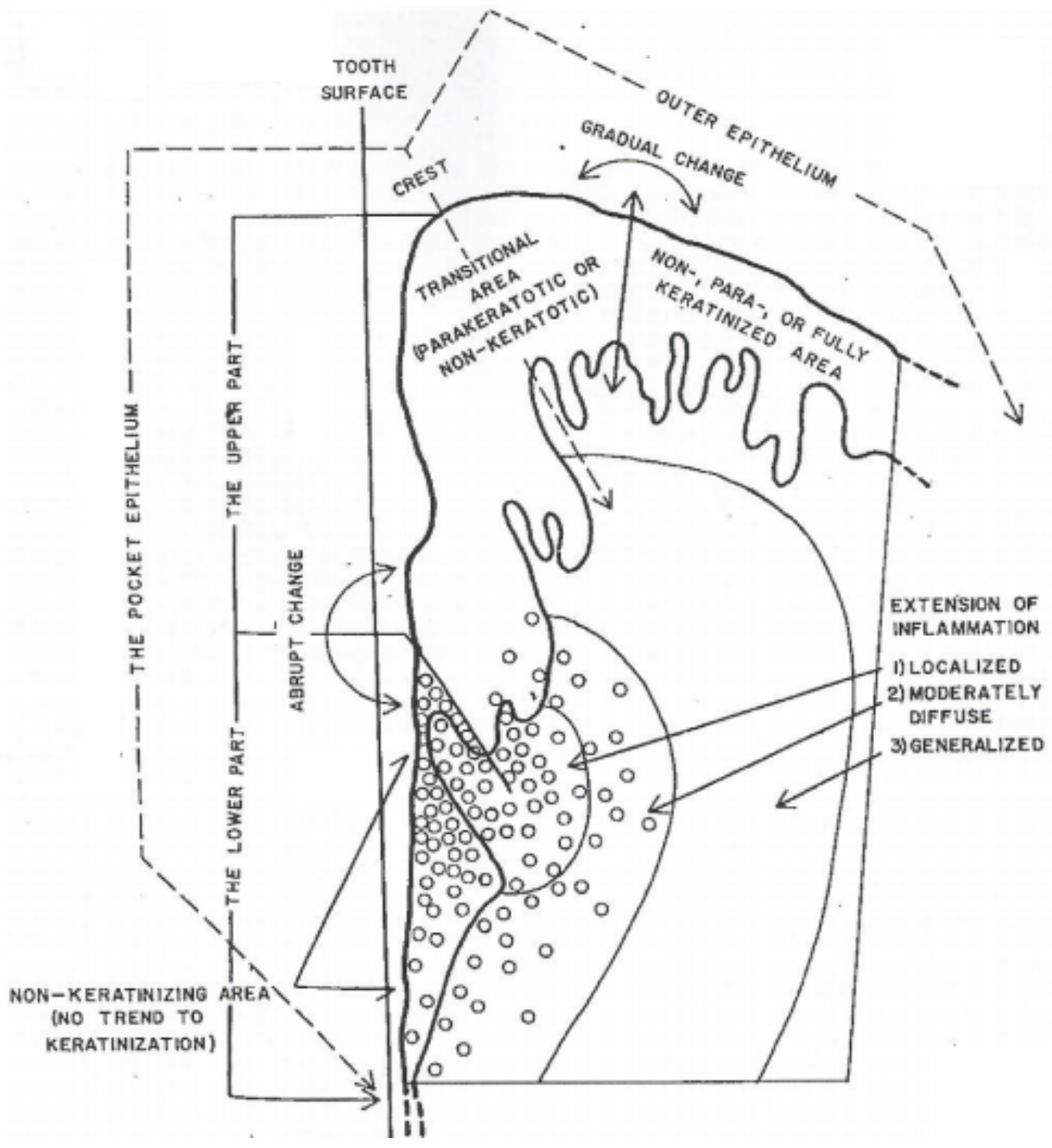

Figure 2. Ultrastructure of plaque-mediated inflammation of the gingiva.

Table 1. Comparing standard sucralfate to polymerized cross-linked sucralfate.

\begin{tabular}{|c|c|c|}
\hline Standard potency sucralfate & \multicolumn{2}{|l|}{ High potency cross-linked polymerized sucralfate } \\
\hline Single molecular sucralfate & Cross-linked sucralfate & Polymerized sucralfate \\
\hline - Hydrated Singular Sucralfate & - Individual sucralfate are Cross-Linked & - Sheets of Sucralfate Hydrogen Bonded \\
\hline $\begin{array}{l}\text { - Singular Sucralfate Molecules } \\
\text { mostly suspended in solution }\end{array}$ & - Cross-linking via bi-/ trivalent cations & - By acidic multi-dentated chelators \\
\hline $\begin{array}{l}\text { - Minimal Amount of Sucralfate } \\
\text { available for coating mucosa }\end{array}$ & $\begin{array}{l}\text { - As Sheets of Sucralfate there is less singular } \\
\text { hydrated sucralfate in solution and more sucralfate is } \\
\text { available for layering. }\end{array}$ & $\begin{array}{l}\text { - Pi-stacking of Sucralfate Sheets } \\
\text { fastening layer by layer }\end{array}$ \\
\hline & - More complete Mucosal Coating & $\begin{array}{l}\text { - } 3 \text { hours following dosing there is } \\
7 \text { fold Greater coating on normal lining } \\
23 \text { fold greater coating on ulcerated lining }\end{array}$ \\
\hline
\end{tabular}

\section{Concentration Dependent Clinical Effect of Sucralfate}

The entire clinical effect of sucralfate is associated with the surface concentration achieved by the administered medicament. When standard formulation sucralfate, whether tablets, suspensions or paste, confront water, the majority of singular sucralfate molecules are hydrated by water, resulting in a reduction of non-hydrated sucralfate available to adhere to the mucosal lining. However, cross-linkage and acid-chelated polymerization, disallows free water dispersion of single sucralfate molecules with resultant random and reduced layering of sucralfate on the mucosal lining. Instead, sucralfate within Orafate, being cross-linked and polymerized present sheets of sucralfate that accumulate upon the mucosal lining via presumed parallel pi-stacking. The results of preferential layering of 'sucralfate sheets' upon each 
McCullough RW (2017) Molecular basis for soft tissue management of gingivitis, periodontitis and peri-implant mucositis using an FDA cleared inflammationtargeting hydrogel - high potency polymerized cross-linked sucralfate - A case series illustrated profile of a drug device (Orafate) and the clinical rationale for use

other, is an increased surface concentration of sucralfate on the mucosal lining throughout the oral cavity. This enhanced surface concentration of sucralfate is prolonged. Three hours following dose administration of Orafate, sucralfate maintains an increased concentration that is $800 \%$ greater than usual on normal lining and $2,400 \%$ greater than usual on ulcerated and inflamed lining [47]. This is illustrated in Table 2 and in Figure 3.

\section{Molecular Mechanism of Action for Orafate Accelerated Healing and Rapid Pain Relief}

Orafate has a device mechanism of action that leads to expedited healing and alleviation of pain. The more efficient compounded layering of polymerized cross-linked sucralfate results in an adherent restrictive micro-environment across the surface to which it is applied. Transiently fixed micro-environs created by adherent HPPCLS facilitate the progression of feed-back controlled-reversal of inflammation. Restrictive micro-environs fashioned by cross-linked layers of polymerized sucralfate "crowds" free-moving anti-inflammatory and pro-inflammatory mediators, indirectly limiting their random movements to "sucralfate-pockets" that overlie mucus gel covered membrane-bound receptors. Spatially limiting the movement of proinflammatory and anti-inflammatory mediators to the vicinity of their respective receptors heightens the chances of receptor site activation. This device action leads to expedited healing.

For the relief of pain, micro-environmental crowding operates similarly. Dental nociceptors for pain are embedded in either the proximal end of dentinal tubules (myelinated A-fibers) or deeper within the dental pulp (non-myelinated C-fibers). Each of these afferent fibers utilize ion-gated switches to turn nociceptors on or off. Restrictive micro-environs fashioned by cross-linked layers of polymerized sucralfate "crowds" and limits free movement of ions required to activate or switch on nociceptors of A-fibers and C-fibers. Denied the free-flux of ions, nociceptors remain neutral and switched off, thereby switching off pain signal.

\section{The Molecular Basis of Orafate Efficacy in Case Series}

In Table 3 the molecular basis of HPPCLS efficacy is discussed using individuals within the case series. The pathophysiology of the signs and symptoms of each patient is explained. Correspondingly, a mode of action for HPPCLS to explain its efficacy is proposed based on the pharmacology of HPPLCS and the pathophysiology of the dental process being addressed. Indeed, larger trials and investigations are required to fully appreciate the potential utility of HPPCLS in dental practice. Yet here in Table 3 are real-world outcomes that support the use of HPPCLS in dental practice. Gingivitis, periodontitis, periimplant mucositis and dentin hypersensitivity, even reversible pulpitis are all inflammatory based disorders solely managed at present with measures that target infection. Only one of the six patients were prescribed an antibiotic (Arestin) which is not a usual occurrence as more often than not, systemic antibiotics are used by non-dental practitioners to address these syndromes. The fact that the clinical problems of most of these patients resolved without antibiotics, speak to the plausibility of having a credible anti-inflammatory program to complement an optimized anti-plaque program. As demonstrated in the case series, targeting inflammation and its molecular process is a viable clinical option for dental practice.

\section{Conclusion}

The foregoing report provided an initial rationale for the clinical use of HPPCLS, in dental practice. By targeting the process

\begin{tabular}{l} 
High Potency Polymerized Cross-linked Sucralfate \\
- Physiochemical Cover \\
- Mechanical Cover \\
- Secondary Effects \\
- Deactivate C-Fiber Voltage-Gated Afferent Neurons \\
- Quiesence of A-fiber firing in Dentinal Tubules \\
- Reinforces the mucus gel \\
\hline
\end{tabular}
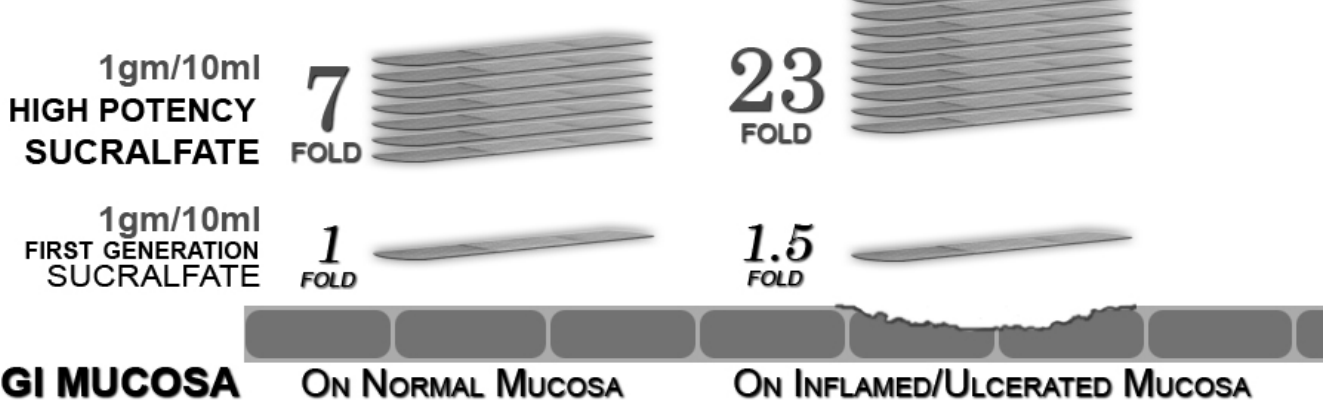

Figure 3. Prolonged exaggerated muco-adherence of polymerized cross-linked sucralfate.

Table 2. Surface concentration of Sucralfate on inflamed (acid injured) \& normal mucosa 3 hour post-dose

\begin{tabular}{|c|c|c|c|}
\hline Pharyngeal and GI Mucosa & $10 \%$ Sucralfate as HPPLCS & $\begin{array}{c}10 \% \text { Standard } \\
\text { Sucralfate Suspension }\end{array}$ & $\begin{array}{c}\text { Fold Increase of HPPCLS Surface } \\
\text { Concentration }\end{array}$ \\
\hline Acid injured GI mucosa & $82.81 \mu \mathrm{g}$ per sq $\mathrm{cm}$ & $3.56 \mu \mathrm{g}$ per sq $\mathrm{cm}$ & 23 fold increase \\
\hline Normal non-injured GI mucosa & $22.01 \mu \mathrm{g}$ per sq $\mathrm{cm}$ & $3.12 \mu \mathrm{g}$ per sq $\mathrm{cm}$ & 7 fold increase \\
\hline Pharynx erosions & $126.24 \mu \mathrm{g}$ per sq $\mathrm{cm}$ & $5.26 \mu \mathrm{g}$ per sq $\mathrm{cm}$ & 24 fold increase \\
\hline Distal esophageal erosions & $93.28 \mu \mathrm{g}$ per sq $\mathrm{cm}$ & $4.24 \mu \mathrm{g}$ per sq $\mathrm{cm}$ & 22 fold increase \\
\hline Gastric mucosal erosions & $82.81 \mu \mathrm{g}$ per sq $\mathrm{cm}$ & $3.56 \mu \mathrm{g}$ per sq $\mathrm{cm}$ & 23 fold increase \\
\hline Colonic mucosal erosions & $94.34 \mu \mathrm{g}$ per sq $\mathrm{cm}$ & $3.87 \mu \mathrm{g}$ per sq $\mathrm{cm}$ & 24 fold increase \\
\hline
\end{tabular}


McCullough RW (2017) Molecular basis for soft tissue management of gingivitis, periodontitis and peri-implant mucositis using an FDA cleared inflammationtargeting hydrogel - high potency polymerized cross-linked sucralfate - A case series illustrated profile of a drug device (Orafate) and the clinical rationale for use

Table 3. Case Series Illustrating Molecular Basis of HPPCLS Clinical Efficacy.

\section{Case Series}

Case 1. Periodontal Pain and Tooth Movement - 52 yo female with long-standing severe periodontitis involving the right maxillary central incisor, its lateral incisor and cuspid treated with scaling, planning and local delivery of antibiotic, developed bleeding, pain and looseness of the 1 st incisor. She was treated with local application of Orafate $(0.8 \mathrm{ml})$ twice daily for 7 days. Pain and bleeding resolved in 2 days. Firmness of tooth restored in 4 days. Anticipated surgical intervention was averted for the time being.

Case 2. Chronic Moderate Periodontal Disease - 56 yo male followed by periodontist for 1 year development of $6 \mathrm{~mm}$ pocket depth involving the left mandibular $1^{\text {st }}$ and $2^{\text {nd }}$ molars with pain and bleeding on probing was treated over the past 12 months with intermittent loca delivery of antibiotics, scaling and planing. He was treated with of Orafate $(0.8 \mathrm{ml})$ and swish and expectorate regimen twice daily for 2 weeks prior to return visit to periodontist. Pocket Depth improved to $4 \mathrm{~mm}$ with no pain or bleeding upon probing. Patient was instructed to continue Orafate daily.

Case 3. Acute Persistent Gingival \& Dental Pain following "Deep Cleaning" - 62 yo female healthy gingiva followed by dentist with biannual dental cleaning for 10 years. Following a session of "deep cleaning" procedure by the dental hygienist, she developed gingival burning and palpable pain, and intermittent bleeding during procedure at the righ mandibular $2^{\text {nd }}$ bicuspid and $1^{\text {st }}$ molar. The bleeding improved but persisted 5 minutes following procedure but gingival burning and palpable tenderness persisted and increased 2 hours post-procedure. There was pain in $2^{\text {nd }}$ bicuspid itself. Treatment with Orafate $(0.8 \mathrm{ml})$, dabbing onto site of pain and bleeding were met with immediate relief of dental pain and of gingival burning with diminished palpable gingival tenderness. The Orafate treatment was repeated in 2 hours. By hour three there was no bleeding and palpable tenderness had stopped. By the sixth hour with no bleeding and no palpable tenderness, there was a faint notice of gingival burning and of bicuspid pain, approximately $1 / 4$ the initial intensity post cleaning. An additional application of Orafate $(0.8 \mathrm{ml})$ was met with immediate cessation of gingival burning and bicuspid pain. Though gingival burning, palpable tenderness and bleeding had completely resolved, patient was advised to brush twice daily with Orafate for 1 week.

Case 4. Peri-implant Mucositis- A 61yo male with a dental implant of the right maxillary central incisor that was 16 years old. He developed peri-implant gingival pain and soreness with scant bleeding and pain on probing. The gingival hard palate at the implant site was treated initially with Orafate $0.8 \mathrm{ml}$ mixed with petroleum jelly $(0.8 \mathrm{ml})$ to facilitated Orafate adherence to peri-implant mucosal every 12 hours. Pain and soreness was relieved by 4 application, but applications were continued for 2 additional days for a total of 4 days. Patient continued twice daily brushing with Orafate with no recurrence of the problem six months from its initial occurrence.

Case 5. Acute Gingivitis - A 62 yo male with Type 2 diabetes with known recurren periogingival mucositis, noted gingival point tenderness, induration and bleeding of the gums during flossing between the left maxillary lateral incisor and cuspid. Normally this episode would last 9-10 days before spontaneously subsiding. At the onset of this episode he was treated with $0.8 \mathrm{ml}$ Orafate mixed with similar quantity of petroleum jelly to the gingiva every 8 hours. Bleeding upon flossing stopped in 1 day, induration improved by $50 \%$ but point tenderness on flossing improved gradually over the next 24 hours with complete resolution of all symptoms by day 3 , less than half the time for spontaneous resolution.

Case 6. Dental Pain to Sweets and Cold Temperature and Pressure Sensitivity - A 63 yo male, with known enamel erosion, and nocturnal teeth grinding, developed 1 week of pain, followed by pressure pain (noted on chewing) and cold temperature sensitivity to his right mandibular $2^{\text {nd }}$ bicuspid which had a fractured, dental caries and in the right maxillary 1 st molar with no previous dental injury. He was advised to brush twice daily with Orafate, starting with the affected teeth. By day 2 of the brushing, pain and cold temperature sensitivity had subsided. By day 4 pressure- pain sensitivity resolved. Two days after stopping Orafate brushing, patient reported return of the pressure pain and faint cold temperature sensitivity. He resumed twice daily brushing with Orafate by dabbing first the affected intradental gingival area then the entire gingival surfaces. Pressure pain and cold sensitivity resolved after 2 days and patient continued Orafate brushing for 1 month.

of inflammation, HPPCLS aids the management of these disorders and may extend the effectiveness of existing therapeutic options, particularly given patients' difficulty with compliance. Improving pocket depths, eliminating post-scaling tooth pain, rapid resolution of gingival mucositis and gingivitis are stated goals of periodontal health. Effective targeting of gingival and periodontal inflammation can only help.

\section{Proposed Explanation of HPPCLS Effect}

HPPCLS Effect in Case 1: Periodontal pain in this case is likely due to both A-fiber from exposed root surfaces and to C-fiber firing due to associated apical inflammation. Looseness of tooth is likely related to periodontal ligament laxity. HPPCLS facilitated the resolution of bothby addressing the molecular processes underlying the clinical signs and symptoms. Dentinal tubule fluid contraction-mediated pain of A-fibers is relieved by HPPCLS mechanical coverage of overlying tubule enamel. This action would also reduce any focal inflammation of the A-fiber innervated pulp. C-fiber mediated pain is relieved by reducing peri-apical inflammation. Integrity of periodontal ligaments maintained by epithelial cell rests of Malassez is undermined by inflammation. HPPCLS targeting inflammation facilitate homeostatic function of cell rests to restore ligament strength.

HPPCLS Effect in Case 2: Deep pockets result from inflammation-mediated recession of the gingivodontal interface at the cervical neck. Gingival inflammation is not purely a one-sided pro-inflammatorydestructive process but a feed-back controlled effort to restore homeostatic barriers involving the presence of anti-inflammatory mediators as well. HPPCLS provide micro-environs that potentiate restorative actions of pro-inflammatory $\&$ anti-inflammatory mediators reversing inflammation in favor of tissue restoration and reduction of pocket depths.

HPPCLS Effect of Case 3: Deep cleaning or scaling can lead to gingival pain as well as increased dental pain. Disruption of gingival tissue create direct exposure of subepithelial tissue to micro-organisms, inciting PAMP $\mathrm{s}$ and DAMPs mediated tissue reaction, with arobust inflammatory response involving neutrophils, monocytes, platelets and macrophages with secondary upregulation of soft tissue afferent neurons. Tooth painin this case is A-fiber mediated and likely due to root exposure of dentinal tubules to hydrostatic fluid and solute changes due to adjacent gingival inflammation with possible diffusionof inflammatory mediators from tissue to tubules. HPPCLS provides mucoadherent and dentocclusive film that immediately stabilizes the hydrodynamics of dentinal tubules resulting in immediate pain relief as A-fiber firing stops. Accelerated gingival healing proceeds due to microenvironmental limitation of mediators to timely engage targeted epithelial receptors

HPPCLS Effect in Case 4: Peri-implant mucositis is immunologically similar to mucositis arising from chemo-radiation. Though caused by bacterial biofilm which require removal, peri-implant mucositis is characterized by a process of feed-back controlled inflammation tasked to restore the physical homeostatic barrier through optimally functioning antimicrobial defenses from gingival epithelium (defensins), $\gamma \delta \mathrm{T}$ cell lymphocytes (cytotoxic mediators) and dendritic cell sampling. HPPCLS maintains an anti-inflammatory cover of the mucositic wound, providing a physically adherent micro-environ conducive to rapid progression of mediator-controlled healing. The best way to prevent peri-implantitis is effective management of peri-implant mucositis. HPPCLS helped in this case.

HPPCLS Effect in Case 5: The gingiva is a periodontal adaptation of the oral mucosa. As such it retains its immunologic means of addressing soft tissue invasion of bacteria utilizing PAMPS, and DAMPs mode of response to restore homeostatic barrier function with anoptimally functioning defensive surveillance system. Complimenting clinician directed anti-plaque measures, HPPCLS targets inflammation, by imposing micro-environmental crowding of mediators of inflammation (including cytotoxins and defensins) into septated areas that accelerate molecular actions programmed to restore homeostasis -which is uninflammed gingival health.

HPPCLS Effect in Case 6: Peripheral dentin is covered by cementum from the cervica area to the roots, but by enamel on the coronal surface. Enamel can be lost by attrition, abrasion (grinding) and erosion (chemical dissolution). Erosion can occur from extrinsic dietary acids (citrus and soda drinks, acidic wines) or intrinsic gastric acids (acid reflux or cyclic vomiting). Relatively exposed dentinal tubules give rise to A-fiber mediated cold and sweets sensitivity from hydrodynamic tubular fluid contractions. Dental caries give rise to exposed dentin with A-fiber-mediated pain. There are five types of dental fractures either one of whichcan lead to dentin hypersensitivity. Pain from pulpitis is mediated by C-fiber as is bite-pressure pain. This case appears to be combination of eroded enamel giving rise to A-fiber pain and reversible $\mathrm{C}$-fiber mediated pulpitis with associatedbite pressure pain. HPPCLS provided stability to dentinal tubular fluid flows, halting A-fiber mediate pain and seep into dental and gingival areas relievingC-fiber mediated pain of reversible pulpitis by reversing pulpal inflammation brought on by dental instability.

The in-office use of HPPCLS following cleaning, with continuance by patient at home, should accelerated healing and should reduce the post procedural gingivodental pain and recurrence of disease. Dentin hypersensitivity affect between $60-98 \%$ of patients with periodontal disease and following periodontal surgery dentin pain predictably increases. The use of HPPLCS with its affect on both A-fiber and C-fiber mediated pain should help such patients as it did in this case series. 
McCullough RW (2017) Molecular basis for soft tissue management of gingivitis, periodontitis and peri-implant mucositis using an FDA cleared inflammationtargeting hydrogel - high potency polymerized cross-linked sucralfate - A case series illustrated profile of a drug device (Orafate) and the clinical rationale for use

Just as HPPCLS can reduce healing of oral mucosal ulcerations (due to chemoradiation) from 84 days to $2-3$ days, it is likely that oral surgical wounds would benefit as well from the use of HPPCLS. Indeed, targeting infection and plaque-borne micro-organisms is the mainstay of periodontal health. Inflammation-targeting therapeutics are an additional approach to managing periodontal health. The commercial availability of HPPCLS (Orafate) now permits dental professionals to simultaneously target the process of inflammation wherever it may occur - the dental pulp, periodontal structures or the gingiva.

\section{References}

1. Löe H (1967) The gingival index, the plaque index and the retention index systems. $J$ Periodontol 38: 610-616. [Crossref]

2. Loe H, Silness J (1963) periodontal disease in pregnancy. I. prevalence and severity. Acta Odontol Scand 21: 533-551. [Crossref]

3. Stamm JW (1986) Epidemiology of gingivitis. J Clin Periodontol 13: 360-366. [Crossref]

4. Li Y, Lee S, Hujoel P, Su M, Zhang W, et al. (2010) Prevalence and severity of gingivitis in American adults. Am J Dent 23: 9-13. [Crossref]

5. Oliver RC, Brown LJ, Loe H (1988) Periodontal diseases in the United States population. J Periodontol 69: 269-278. [Crossref]

6. Zitzmann NU, Berglundh T (2008) Definition and prevalence of peri-implant diseases. J Clin Periodontol 35: 286-291. [Crossref]

7. Lindhe J, Meyle J (2008) Peri-implant diseases: Consensus report of the sixth European workshop on periodontology. J Clin Periodontol 35: 282-228. [Crossref]

8. Roos-Jansaker AM, Lindahl C, Renvert H, Renvert S (2006) Nine -to- fourteen yeart follow up of implant treatment. Part II: Presence of peri-implant lesions. J Clin Periodontol 33: 290-295. [Crossref]

9. Wilson TG Jr (1996) Compliance and its role in periodontal therapy. Periodontol 2000 12: 16-23. [Crossref]

10. Wilson TG Jr. (1996) Supportive periodontal treatment introduction-definition, extent of need, therapeutic objectives, frequency and efficacy. Periodontol 2000 12: 11-15. [Crossref]

11. Drisko CH (2001) Nonsurgical periodontal therapy. Periodontol 2000 25: 77-88. [Crossref]

12. Zeza B, Pilloni A (2012) Peri-implant mucositis treatments in humans: a systematic review. Ann Stomatol (Roma) 3: 83-89. [Crossref]

13. Renert S, Roos-Jansaker AM, Claffey AM (2008) Non-surgical treatment of periimplant mucositis and peri-implantitis: a literature review. J Clin Periodontol 35: 305315. [Crossref]

14. Salvi GE, Aglietta M, Eick S, Sculean A, Lang NP, Rameier CA (2012) Reversibility of experimental peri-inmplant mucositis compared with experimental gingivitis in humans. Clin Oral Impl Res 23: 182-190. [Crossref]

15. Cutler CW, Stanford TW, Abraham C, Cederberg RA, Boardman TJ, Ross C (2000) Clinical benefits of oral irrigatin for periodontitis are related to reduction of proinflammatory cytokine levels and plaque. J Clin Periodontol 27: 134-143. [Crossref]

16. Aljateeli M, Koticha T, Bashutski J, Sugai JV, Braun TM, et al. (2014) Surgical periodontal therapy with and without initial scaling and root planing in the management of chronic periodontitis: a randomized clinical trial. J Clin Periodontol 41: 693-700. [Crossref]

17. Bar-Shalom, Bukh N, Hamberger J. Method of treating conditions of teeth and their supporting tissue with sucralfate. US Patent 5,240,710. Aug 31, 1993 and US Patent 5,709.873. Jan 20, 1998 .

18. McCullough RW (2015) Single agent anti-mucositis protocol for oral and gastrointestinal mucositis and other implications on current concepts regarding chemoradiation induced mucositis and its management from a phase IV post-market surveillance of ProThelial - high potency polymerized cross-linked sucralfate (HPPCLS). Eur J Oncol Pharm 9: $1-11$.

19. McCullough RW (2015) A 66 patient multi-institutional phase IV post-authorization surveillance of ProThelial (high potency polymerized cross-linked sucralfate)- Single agent efficacy for the Prevention and Rapid reversal of Chemo-radiation induced oral, esophageal and intestinal mucositis. Brit J Med Med Research 10: 1-17.
20. Stewart PS (1996) Theoretical aspects of antibiotic diffusion into microbial biofilms. Antimicrob Agents Chemother 40: 2517-2522. [Crossref]

21. Stewart PS, Costerton JW (2001) Antibiotic resistance of bacteria in biofilms. Lance 358: 135-138. [Crossref]

22. Noyan U, Yilmaz S, Kuru B, Kadir T, Acar O, Büget E (1997) A clinical and microbial evaluation of systemic and local metronidazole delivery in adult periodontitis patients. J Clin Periodontol 24: 158-165. [Crossref]

23. Rodrigues RM, Goncalves C, Souto R, Feres-Filho EJ, Uzeda M, et al. (2004) Antibiotic resistance profile of the subgingival microbiota following systemic or local tetracycline. J Clin Periodontal 31: 420-427.

24. Moter A, Kovacevic D, Mallmann C, Göbel UB (2005) Sensitive FISH: fluorescence in situ hybridization in microbiological diagnosis. Pneumologie 59: 425-427. [Crossref]

25. Veeh RH, Shirtliff ME, Petik JR, Flood JA, Davis CC, et al. (2003) Detection of staphylococcus aureus bioflilm on tampons and menses components. $J$ Infect Dis 188 519-530. [Crossref]

26. Wecke J, Kersten T, Madela K, Moter A, Göbel UB, et al. (2000) A novel technique for monitoring the development of bacterial biofilms in human periodontal pockets. FEMS Microbiol Lett 191: 95-101. [Crossref]

27. Listgarten MA (1976) Structure of the microbial flora associated with periodontal health and disease in man: a light and electron microscopic study. J Periodontol 47 : 1-18. [Crossref]

28. Newman MG, Socransky SS, Savitt ED, Propas DA, Crawford A (1976) Studies of the microbiology of periodontosis. J Periodontol 47: 373-379. [Crossref]

29. Jain N, Gupta A, Menna N (2013) An insight into neurophysiology of pupal pain: facts and hypothesis. Korean J Pain 26: 347-355. [Crossref]

30. Pashley DH, Tay FR (2008) Dentin hypersensitivity: current state of the art and science. Dentin Hypersensitivity Consensus Monograph 4: 1-11.

31. Xiong J, Gronthos S, Barthod PM (2013) Role of the epithelial cell rests of Malassez in the development, maintenance and regeneration of periodontal ligament tissues. Periodontology 2000 63: 217-233. [Crossref]

32. Bosshardt DD, Selvig KA (1997) Dental cementum: the dynamic tissue covering of the root. Periodontol 2000 13: 41-75. [Crossref]

33. Takarada H, Canttoni M, Sugimoto A, Rose GG (1974) Ultrastructural studies of human gingiva. I. The upper part of the pocket epithelium in chronic periodontitis. $J$ Periodontol 45: 30-42. [Crossref]

34. Takarada H, Canttoni M, Sugimoto A, Rose GG (1974) Ultrastructural studies of human gingiva. II. The lower part of the pocket epithelium in chronic periodontitis. $J$ Periodontol 45: 155-169. [Crossref]

35. Takarada H, Canttoni M, Sugimoto A, Rose GG (1974) Ultrastructural studies of human gingiva. III. Changes in the basal lamina in chronic periodontis. J Periodontol 45: 288-302. [Crossref]

36. Marshall RI (2004) Gingival defensins: linking the innate and adaptive immune responses to dental plaque. Periodontol 2000 35: 14-20. [Crossref]

37. Greer A, Zenobia C, Darveau RP (2013) Defensins and LLL-37: a review of function in the gingival epithelium. Periodontol 2000 63: 67-79. [Crossref]

38. Benakanakere M, Kinane DF (2012) Innate cellular responses to the periodontal biofilm. Front Oral Biol 15: 41-55. [Crossref]

39. da Motta RJ, Tirapelli C, Juns da Silva R, Villafuerte KR, Almeida LY, et al. (2016) Immature, but not mature, dendritic cells are more often present in aggressive periodontitis than chronic periodontitis: An immunohistochemical study. J Periodontol 87: 1499-1507. [Crossref]

40. Havran WL, Jameson JM Witherden DA (2005) Epithelial cells and their neighbors II Interactions between intraepithelial lymphocytes and neighboring epithelial cells. $\mathrm{Am} J$ Physiol Gastrointest Liver Physiol 289: G627-G630. [Crossref]

41. Lundqvist C, Baranov V, Teglund S. Hammarstrom S, Hammarstom ML (1994) Cytokine profile and ultrastructure of intraepithelial gamma delta $\mathrm{T}$ cells in chronically inflamed human gingiva suggest a cytotoxic effector function. $J$ Immunol 153: 23022312. [Crossref]

42. Lundqvist C, Baranov V, Söderström K, Athlin L, Kiessling R, et al. (1995) Phenotype and cytokine profile of intraepithelial lymphocytes in human small and large intestine. Ann N Y Acad Sci 756: 395-399. [Crossref]

43. Silhavy TJ, Kahne D, Walker S (2010) The bacterial cell envelope. Cold Spring Harb Perspect Biol 2: a000414. [Crossref] 
McCullough RW (2017) Molecular basis for soft tissue management of gingivitis, periodontitis and peri-implant mucositis using an FDA cleared inflammationtargeting hydrogel - high potency polymerized cross-linked sucralfate - A case series illustrated profile of a drug device (Orafate) and the clinical rationale for use

44. Land WG (2015) The role of damage-associated molecular patterns in human disease. Part 1 - promoting inflammation and immunity. Sultan Qaboos Univ J 15: 9-21. [Crossref]

45. McGaughey GB, Gagné M, Rappé AK (1998) pi-Stacking interactions. Alive and well in proteins. J Biol Chem 273: 15458-15463. [Crossref]
46. Ma JC, Dougherty DA (1997) The Cationminus signpi Interaction. Chem Rev 97: 13031324. [Crossref]

47. Kashimura K, Ozawa K (1999) Sucralfate preparations. US Patent 5,968,906. Oct 19, 1999.

Copyright: (C2017 McCullough RW. This is an open-access article distributed under the terms of the Creative Commons Attribution License, which permits unrestricted use, distribution, and reproduction in any medium, provided the original author and source are credited. 